\title{
A Converse Lyapunov Theorem for Switched Linear Systems with Dwell Times
}

\author{
Fabian Wirth*
}

\begin{abstract}
We study linear switching systems, where timevariations have to satisfy restrictions on the dwell time, that is on the minimum distance between discontinuities. The main objective of the paper is to construct parameter dependent Lyapunov functions, which characterize the exponential growth rate. This is possible in the generic irreducible case.

Index Terms-converse Lyapunov theorem, linear parameter varying systems, linear switching systems, linear flows on vector bundles, periodic systems.
\end{abstract}

\section{INTRODUCTION}

This paper is a reduction of the paper [1] to a content that can be presented within the given space limitations. For full details we refer to the journal paper, especially all omitted proofs can be found there. In a few points the results of [1] are extended.

In [1] we consider linear time-varying systems of the form

$$
\dot{x}(t)=A(t) x(t),
$$

where $A: \mathbb{R} \rightarrow \mathcal{M}$ is a measurable map, and $\mathcal{M}$ is a compact set of real or complex matrices of a given dimension. We are interested not in one individual system, but in the exponential growth rate of a set of systems, that is described by a subset $\mathcal{A} \subset L^{\infty}(\mathbb{R}, \mathcal{M})$. The stability and spectral properties of such kinds of systems have been actively investigated over the past two decades.

In particular in [1] a framework covering many of the systems studied in the areas of linear parameter varying systems with constraints on the derivative and of linear switching systems with dwell times. A class of linear time-varying systems is introduced, that allows for (i) bounds on the minimal time between discontinuities and (ii) bounds on the derivative of parameter variations between discontinuities.

Here we will deal with a far less general case, that is a general case of [1]. Consider a finite set of matrices $\mathcal{A}:=\left\{A_{1}, \ldots, A_{m}\right\} \subset \mathbb{R}^{n \times n}$ and a dwell-time $h>0$. The switched linear system $\Sigma=(\mathcal{A}, h)$ is defined as the family of time-varying systems given by

$$
\dot{x}(t)=A_{u(t)} x(t),
$$

where $u: \mathbb{R}_{+} \rightarrow\{1, \ldots, m\}$ is a piecewise continuous map, with the property, that the discontinuities of $u$, denoted by $0<t_{0}<t_{1}<t_{2} \ldots$ satisfy the dwell time condition

$$
t_{k+1}-t_{k} \geq h, \quad \forall k \geq 1 .
$$

In other words, discontinuities of $u$ are at least distance $h$ apart. We refer to $u$ as a switching signal. Without loss of

* Hamilton Institute, NUI Maynooth, Maynooth, Co. Kildare, Ireland Fabian.Wirthenuim.ie generality, we will assume switching signals to be rightcontinuous. The set of switching signals is denoted by $\mathcal{U}:=\left\{u: \mathbb{R}_{+} \rightarrow\{1, \ldots, m\} \mid\right.$ right-continuous, piecewise continuous, with discontinuities $h$ apart. $\}$.

For fixed $u \in \mathcal{U}$ the linear time-varying system (2) generates an evolution operator, which we will denote by $\Phi_{u}(t, s), t, s \geq 0$. We are interested in stability properties with respect to all switching signals $u \in \mathcal{U}$.

The main contribution of the present paper lies in the construction of Lyapunov functions parameterized by $i \in$ $\{1, \ldots, m\}$, that characterize the exponential growth rate of the system under consideration. The construction is possible in the generic irreducible case, in which the system leaves no nontrivial subspace invariant. For each parameter the corresponding Lyapunov function is a norm. One of the features of the Lyapunov functions is, that for any solution the corresponding infinitesimal decay is upper bounded by the maximal growth rate. Also the exponential growth rate can be realized instantaneously from every initial condition of state and parameter. As in [2] it would be possible to consider smooth approximations to obtain differentiable Lyapunov functions, which still yield a decay arbitrarily close to the growth rate. This problem is not pursued here, as the method is well described in the literature, see [2], [3], [4].

Using the existence of Lyapunov functions, a fairly simple proof of a version of the Gelfand formula can be given, see [1], [5]. By this result the exponential growth rate can be approximated to arbitrary precision using periodic parameter variations. In [6] results on the Lipschitz continuous dependence of the growth rate of the data are obtained using the existence of Lyapunov functions constructed here.

Papers concerned with linear differential inclusions and families of time-varying systems often treat the case of linear inclusions (or (2) with arbitrary measurable switching signals) which amounts to taking $\mathcal{U}=L^{\infty}(\mathbb{R}, \mathcal{M})$. In this area a detailed description of spectral concepts is available, see [7], [8], [9] and a good Lyapunov theory has been developed [10], [11], [12].

For an overview and much of the related literature in the area of switched systems we refer to [13], [14], [15]. The approach to consider switched systems with a dwell time condition derives its motivation in part from adaptive control and has been discussed in [16], [17]. Sufficient conditions for the existence of Lyapunov functions in terms of LMIs are available, see e.g. [18].

We proceed as follows. In the ensuing Section II we introduce the exponential growth rate under the (essential) 
assumption of shift invariance. This is the quantity of interest in this paper. In Section IV irreducibility of a system is introduced and some immediate consequences of this property are shown. The assumption of irreducibility is used in Section V to construct parameter dependent Lyapunov norms, that characterize the exponential growth rate. We particularly discuss the case of linear switching systems with dwell time, for which an easy interpretation is available.

Finally, we would like to warn the reader that our use of the term Lyapunov function is not quite the standard one. It will be used to denote functions that characterize the exponential growth rate of the system if evaluated along trajectories. Now if the system is stable, then this will give the usual decrease condition. However, if the system is not exponentially stable, then we still speak of a Lyapunov function because of the characterization of the growth rate.

\section{Exponential GROWth RATES}

We now define the object of interest in this paper which is the (uniform) exponential growth rate associated to system (2). Given the map $\mathcal{A}$ and $h>0$ and define for $t \geq 0$ the sets of finite time evolution operators

$\mathcal{S}_{t}(\mathcal{A}, h):=\left\{\Phi_{u}(t, 0) \mid u \in \mathcal{U}\right\}, \quad \mathcal{S}(\mathcal{A}, h):=\bigcup_{t \geq 0} \mathcal{S}_{t}(A, \mathcal{U})$

We now introduce for $t>0$ finite time growth constants given by

$$
\widehat{\rho}_{t}(\mathcal{A}, h):=\sup \left\{\frac{1}{t} \log \|S\| \mid S \in \mathcal{S}_{t}(\mathcal{A}, h)\right\} .
$$

It is easy to see, that the function $t \longmapsto t \widehat{\rho}_{t}(\mathcal{A}, h)$ is subadditive. Using a folklore result (see e.g. [19, p. 27/28]) this implies, that the following limit exists

$$
\widehat{\rho}(\mathcal{A}, h):=\lim _{t \rightarrow \infty} \widehat{\rho}_{t}(\mathcal{A}, h)=\inf _{t \geq 0} \widehat{\rho}_{t}(\mathcal{A}, h) .
$$

It is well known, that an alternative way to describe $\hat{\rho}$ is given by

$$
\begin{aligned}
\widehat{\rho}(\mathcal{A}, h)= & \inf \{\beta \in \mathbb{R} \mid \exists M \geq 1 \text { such that } \\
& \left.\left\|\Phi_{u}(t, 0)\right\| \leq M e^{\beta t} \text { for all } u \in \mathcal{U}, t \geq 0\right\} .
\end{aligned}
$$

For this reason the quantity $\hat{\rho}(\mathcal{A}, h)$ is called uniform exponential growth rate of the family of switched linear system (2). An alternative way to define exponential growth is to employ a trajectory-wise definition. In this case we define the Lyapunov exponent corresponding to an initial condition $x_{0} \in \mathbb{R}^{n} \backslash\{0\}$ and $u \in \mathcal{U}$ by

$$
\lambda\left(x_{0}, u\right):=\limsup _{t \rightarrow \infty} \frac{1}{t} \log \left\|\Phi_{u}(t, 0) x_{0}\right\|
$$

and define as exponential growth rate $\kappa(\mathcal{A}, h):=$ $\sup \left\{\lambda(x, u) \mid 0 \neq x \in \mathbb{R}^{n}, u \in \mathcal{U}\right\}$. It follows using Fenichel's uniformity lemma that $\kappa(\mathcal{A}, h)=\hat{\rho}(\mathcal{A}, h)$, see [1] and [9, Prop. 5.4.15].

One might now be tempted to look for norms that are Lyapunov functions for the whole system and characterize the quantity $\hat{\rho}(\mathcal{A}, h)$ as in the case of linear differential inclusions, see [10], [12]. However, the following lemma shows, that this is not a very fruitful enterprise.

Lemma 2.1: Consider a switched system $(\mathcal{A}, h)$. If there is a norm $v$ on $\mathbb{R}^{n}$, such that for all $x \in \mathbb{R}^{n}, u \in \mathcal{U}$ and the corresponding evolution operator $\Phi_{u}(t, s)$ it holds, that

$$
v\left(\Phi_{u}(t, 0) x\right) \leq e^{\hat{\rho}(\mathcal{A}, h) t} v(x), \quad \forall t \geq 0,
$$

then $\hat{\rho}(\mathcal{A}, h)=\rho$, where

$\rho:=\max \left\{\lambda(x, B) \mid 0 \neq x \in \mathbb{R}^{n}, B: \mathbb{R} \rightarrow \mathcal{A}\right.$ measurable $\}$

and where $\lambda(x, B)$ denotes the Lyapunov exponent corresponding to the initial condition $x$ and $B$ defined as in (6).

By the previous lemma, a norm satisfying (7) can only exist for (2), if the parameter varying system realizes the exponential growth, which is obtained by allowing all measurable functions with values in $\mathcal{A}$; in other words, by studying (2) with $\mathcal{U}=L^{\infty}(\mathbb{R}, \mathcal{A})$. For general switched systems with dwell time this situation is rarely encountered. For this reason we use a different approach, that introduces a family of norms with an extremal property. The idea to use parameter dependent Lyapunov functions, proposed by several authors (see e.g. [20], [18]), can be made exact in this way. That is, a family of parameter dependent Lyapunov norms may be constructed, such that the exponential growth rate of system (2) is the incremental growth rate with respect to this family. Note that we cannot restrict our attention to quadratic norms to perform such a construction.

Remark 2.1: The main technical problem in this paper is, that $\mathcal{S}(\mathcal{A}, h)$ does not naturally carry the structure of a semigroup. This complicates matters compared to the case of linear inclusions of the form

$$
\dot{x} \in\{A x \mid A \in A(\Theta)\},
$$

as studied in [10], [7], [13], [21], [12] and references therein.

\section{Concatenation of Switching Signals}

As we will be dealing with set-valued maps, let us briefly recall, that a set-valued map $F$ from $X \subset \mathbb{R}^{m}$ to $\mathbb{R}^{n}$ is a map, that associates to every point in $X$ a subset of $\mathbb{R}^{n}$. We will only encounter the easy case, in which the images are compact sets. Such a map is called locally Lipschitz continuous, if for every compact subset $K \subset X$ there is a constant $L$, such that $d_{H}(F(x), F(y))<L\|x-y\|$ for all $x, y \in K$.

In this section we assume the system $\Sigma=(\mathcal{A}, h)$ to be given. For ease of notation we will therefore suppress the dependence of $\hat{\rho}, \mathcal{S}_{t}$, etc. on these data. In general, concatenation of admissible switching signals does in general not result in an admissible switching signal. Given two switching signals $u, w \in \mathcal{U}$ we define the concatenation at time $t$ by

$$
\begin{gathered}
\left(u \diamond_{t} w\right)(s):=\left\{\begin{array}{ll}
u(s), & s<t \\
w(s-t), & t \leq s
\end{array} .\right. \\
\mathcal{U}(i):=\left\{w \in \mathcal{U} \mid w(0)=i \text { or } t_{0}(w) \geq h\right\} .
\end{gathered}
$$

(Recall, that $t_{0}>0$ is the first discontinuity of $w$, possibly $t_{0}=\infty$.) Thus the set $\mathcal{U}(i)$ denotes the set of switching 
signals that may be concatenated at time $t$ to a switching signal $u$ with the properties that $u\left(t^{-}\right)=i$ and so that the largest discontinuity $t^{*}$ of $u$ smaller than $t$ satisfies $t-t^{*} \geq h$. In this case we can either use a continuous extension by $i$ and there is no restriction on the next discontinuity, or we can introduce a discontinuity at $t$ by switching to $j \neq i$ in which case another time span of length $h$ has to elapse before a further discontinuity can occur.

For each $i \in\{1, \ldots, m\}$ and $t \geq 0$ we define the set of evolution operators "starting in $i$ " by

$$
\mathcal{S}_{t}(i):=\left\{\Phi_{u}(t, 0) \mid u \in \mathcal{U}(i)\right\} .
$$

Similarly, we define for $i, j \in\{1, \ldots, m\}$ and for $t \geq h$ the sets of evolution operators "starting in $i$ and ending at $j$ " by

$$
\begin{aligned}
\mathcal{R}_{t}(i, j) & :=\left\{\Phi_{u}(t, 0) \mid u \in \mathcal{U}(i),\right. \text { and for all } \\
w & \left.\in \mathcal{U}(j) \text { it holds that } u \diamond_{t} w \in \mathcal{U}(i)\right\} .
\end{aligned}
$$

Thus by definition if $R \in \mathcal{R}_{s}(i, j)$ and $S \in \mathcal{S}_{t}(j)$, then $S R \in \mathcal{S}_{t+s}(i)$. We now define

$$
\mathcal{S}_{\leq T}(i):=\bigcup_{0 \leq t \leq T} \mathcal{S}_{t}(i) \text { and } \mathcal{S}(i):=\bigcup_{t \geq 0} \mathcal{S}_{t}(i),
$$

and in a similar manner $\mathcal{R}_{\leq T}(i, j)$ as well as $\mathcal{R}(i, j)$. Note that for every $i \in\{1, \ldots, m\}$ the set $\mathcal{R}(i, i)$ is a semigroup.

Remark 3.1: Note that for any $i, j \in\{1, \ldots, m\}$ the set $\mathcal{R}_{t}(i, j), t \geq h$ is not empty. It suffices to define $u(s)=$ $j, 0 \leq s \leq t$ to obtain an element of the set.

In a first step let us clarify the continuity properties of the sets just defined. To this end we note the following consequence of the Arzela-Ascoli theorem.

Lemma 3.1: Let $\mathcal{A}$ and $h>0$ be fixed and consider the space $\mathcal{U}$ of admissible switching signals.

Given $T \geq h$ and sequences $i_{k}, j_{k} \in\{1, \ldots, m\}, u_{k} \in$ $\mathcal{U}\left(i_{k}\right)$ with $\Phi_{u_{k}}(T, 0) \in \mathcal{R}\left(i_{k}, j_{k}\right)$, there exist subsequences, such that

(i) the limits $\lim _{\mu \rightarrow \infty} i_{k_{\mu}}=: i$ and $\lim _{\mu \rightarrow \infty} j_{k_{\mu}}=: j$ exist, (ii) $\left\{u_{k_{\mu}}\right\}_{\mu \in \mathbb{N}}$ converges in the weak-*-topology on $[0, T]$ to an admissible switching signal $u \in \mathcal{U}(i)$ with $\Phi_{u}(T, 0) \in$ $\mathcal{R}(i, j)$.

Furthermore,

$$
\Phi_{u_{k_{\mu}}}(t, 0) \rightarrow \Phi_{u}(t, 0), \text { uniformly on }[0, T] .
$$

We are now ready to prove an essential though fairly basic lemma concerning the dependence of the parameterized sets of transition operators on time and the parameters.

Lemma 3.2: Consider system (2). Then

(i) For all $(t, i, j) \in[0, \infty) \times\{1, \ldots, m\}^{2}$ the sets $\mathcal{S}_{t}(i)$ and $\mathcal{R}_{t}(i, j)$ are compact.

(ii) The maps $\mathcal{S}: \mathbb{R}_{+} \times\{1, \ldots, m\} \rightarrow \mathcal{K}\left(\mathbb{R}^{n}\right), \mathcal{R}:[h, \infty) \times$ $\{1, \ldots, m\}^{2} \rightarrow \mathcal{K}\left(\mathbb{R}^{n}\right)$ given by

$$
(t, i) \mapsto \mathcal{S}_{t}(i), \quad(t, i, j) \mapsto \mathcal{R}_{t}(i, j)
$$

are locally Lipschitz continuous.

(iii) If $\mathcal{S}(A, \mathcal{U})$ is bounded, then the Lipschitz constants with respect to $i \in\{1, \ldots, m\}$ (resp. $(i, j) \in\{1, \ldots, m\}^{2}$ ) may be chosen uniformly in $t$.
If we want to describe the exponential growth rate within the subsets of evolution operators with given initial and end condition, this leads to the definitions

$$
\begin{array}{r}
\widehat{\rho}_{t}(i):=\max \left\{\frac{1}{t} \log \|S\| \mid S \in \mathcal{S}_{t}(i)\right\}, \\
\widehat{\rho}_{t}(i, j):=\max \left\{\frac{1}{t} \log \|S\| \mid S \in \mathcal{R}_{t}(i, j)\right\} .
\end{array}
$$

With this the problem arises, that the functions $t \longmapsto t \widehat{\rho}_{t}(i)$, and $t \longmapsto t \widehat{\rho}_{t}(i, j)$ are no longer subadditive, so that it does not follow automatically to what value they are converging, if at all. It is therefore useful to point out the following.

Lemma 3.3: Consider the system (2). There is a constant $C \in \mathbb{R}$, such that for all $i, j \in\{1, \ldots, m\}$ we have, that

$$
t \widehat{\rho}_{t}(i, j) \geq t \hat{\rho}-C, \forall t>0 .
$$

In particular, it follows for all $i, j \in\{1, \ldots, m\}$, that

$$
\widehat{\rho}=\lim _{t \rightarrow \infty} \widehat{\rho}_{t}(i, j)=\lim _{t \rightarrow \infty} \widehat{\rho}_{t}(i) .
$$

\section{IRREDUCIBILITY}

We aim to construct parameter dependent Lyapunov functions that exactly reflect the exponential growth rate of the system $\Sigma=(\mathcal{A}, h)$. To this end it is crucial to assume the irreducibility of $\mathcal{A}$. Recall that a set of matrices $\mathcal{M} \subset \mathbb{R}^{n \times n}$ is called irreducible, if only the trivial subspaces $\{0\}$ and $\mathbb{R}^{n}$ are invariant under all $A \in \mathcal{M}$ and reducible otherwise.

Remark 4.1: (i) Note that if $m \geq 2$ the set of irreducible sets $\mathcal{A}$ is open and dense in the set of finite sets of matrices with at most $m$ elements in any standard metric, e.g. the Hausdorff metric.

(ii) If $\mathcal{A}$ is reducible, we can find a similarity transformation $T$, such that for all $j \in\{1, \ldots, m\}$ the transformed matrix $T A_{j} T^{-1}$ is of the form

$$
\left[\begin{array}{cccc}
A_{11}(j) & A_{12}(j) & \ldots & A_{1 d}(i) \\
0 & A_{22}(j) & \ldots & A_{2 d}(j) \\
& \ddots & \ddots & \vdots \\
0 & & 0 & A_{d d}(j)
\end{array}\right]
$$

where the sets $\left\{A_{i i}(j) \mid j \in\{1, \ldots, m\}\right\} \subset \mathbb{R}^{n_{i} \times n_{i}}$ are irreducible or $\{0\}, i=1, \ldots, d$. It is an easy exercise to show, that in this case $\hat{\rho}(\mathcal{A}, h)=\max _{i=1, \ldots, d} \hat{\rho}\left(\mathcal{A}_{i}, h\right)$, where $\mathcal{A}_{i}$ is the set of matrices obtained by taking the $i$ th diagonal block. Having said this it is clear, that for the analysis of $\hat{\rho}$ with respect to one system we can assume irreducibility without loss of generality.

The next simple lemma, see [12, Lemma 3.1], is crucial in the following construction.

Lemma 4.1: Let $\mathcal{S} \subset \mathbb{R}^{n \times n}$ be an irreducible semigroup. For any family of sets $\mathcal{S}_{t}, t \in \mathbb{R}_{+}$with

$$
\mathcal{S}=\bigcup_{t \geq 0} \mathcal{S}_{t}
$$

there are $\varepsilon>0$ and $T \in \mathbb{R}_{+}$, such that for all $z \in \mathbb{R}^{n}, A \in$ $\mathbb{R}^{n \times n}$ there is an $S \in \bigcup_{1 \leq t \leq T} \mathcal{S}_{t}$ with

$$
\|A S z\| \geq \varepsilon\|A\|\|z\| \text {. }
$$


We now begin to study the consequences of irreducibility. The following properties are essential in our construction of Lyapunov functions.

Proposition 4.1: Consider system (2). If $\mathcal{A}$ is irreducible, then for all $i, j \in\{1, \ldots, m\}$

(i) the set $\mathcal{R}(i, j)$ is irreducible,

(ii) the set $\mathcal{S}(i)$ is irreducible.

Proof: (i) Fix an arbitrary nontrivial subspace $X$ and let $\Phi_{u}(t, 0) \in \mathcal{R}(i, j)$ with $t \geq h$ be such that $\Phi_{u}(t, 0) X=X$. (If no such $\Phi$ exists we are done.) Let $t^{*} \in(0, t)$ be a discontinuity of $u$, or if such a discontinuity does not exist let $t^{*}=t / 2$. Denote $Y:=\Phi_{u}\left(t^{*}, 0\right) X$. As $\mathcal{A}$ is irreducible, there exists a $i^{*} \in\{1, \ldots, m\}$, such that $\exp \left(A\left(i^{*}\right) s\right) Y \not \subset Y$ for some $s \geq h$. Hence $\Phi_{u}\left(t, t^{*}\right) \exp \left(A\left(i^{*}\right) s\right) \Phi_{u}\left(t^{*}, 0\right) X \not \subset$ $X$. On the other hand $\Phi_{u}\left(t, t^{*}\right) \exp \left(A\left(i^{*}\right) s\right) \Phi_{u}\left(t^{*}, 0\right) \in$ $\mathcal{R}(i, j)$, because we may at time $t^{*}$ switch to $A\left(i^{*}\right)$, remain there for the time $s$, and switch back to $u\left(t^{*}\right)$. This defines an admissible switching signal; and the assertion follows.

(ii) This is immediate from (i) as $\mathcal{S}(i)=$ $\cup_{j \in\{1, \ldots, m\}} \mathcal{R}(i, j)$.

\section{PARAMETERIZEd LyAPUNOV FUNCTIONS}

In this section the main result of the paper is derived. In Theorem 5.1 we obtain the existence of parameterized Lyapunov functions that characterize the exponential growth rate.

The main step of the proof relies on the following construction. By Lemma 3.3 the exponential growth in $\mathcal{S}$ and in the subsets $\mathcal{S}(i), \mathcal{R}(i, \eta)$ is essentially the same. It therefore makes sense to define limit sets as follows.

$$
\begin{gathered}
\mathcal{S}_{\infty}(i):=\left\{S \in \mathbb{R}^{n \times n} \mid \exists t_{k} \rightarrow \infty, S_{k} \in \mathcal{S}_{t_{k}}(i):\right. \\
\left.e^{-\hat{\rho} t_{k}} S_{k} \rightarrow S\right\} . \\
\mathcal{R}_{\infty}(i, j):=\left\{S \in \mathbb{R}^{n \times n} \mid \exists t_{k} \rightarrow \infty, S_{k} \in \mathcal{R}_{t_{k}}(i, j):\right. \\
\left.e^{-\hat{\rho} t_{k}} S_{k} \rightarrow S\right\} .
\end{gathered}
$$

We note the following properties of $\mathcal{S}_{\infty}(i)$ and $\mathcal{R}_{\infty}(i, j)$. Lemma 5.1: Consider the system (2). If $\mathcal{A}$ is irreducible then

(i) the set $\cup_{i \in\{1, \ldots, m\}} \mathcal{S}_{\infty}(i)$ is bounded, and for all $i, j \in\{1, \ldots, m\}$ it holds that

(ii) $\mathcal{R}_{\infty}(i, j)$ is a compact, nonempty set not equal to $\{0\}$,

(iii) $\mathcal{S}_{\infty}(i)$ is a compact, nonempty set not equal to $\{0\}$,

(iv) for every $t \geq 0$ we have that, if $R \in \mathcal{R}_{t}(i, j)$ and $S \in \mathcal{S}_{\infty}(j)$, or if $R \in \mathcal{R}_{\infty}(i, j)$ and $S \in \mathcal{S}_{t}(j)$, then $e^{-\hat{\rho} t} S R \in \mathcal{S}_{\infty}(i)$,

(v) for every $S \in \mathcal{S}_{\infty}(i), t \geq 2 h$ there exist $j \in\{1, \ldots, m\}$, $R \in \mathcal{R}_{t}(i, j)$, and $T \in \mathcal{S}_{\infty}(j)$, such that $S=e^{-\hat{\rho} t} T R$,

(vi) $\mathcal{R}_{\infty}(i, i), \mathcal{S}_{\infty}(i)$ are irreducible.

Proof: Without loss of generality we may assume that $\hat{\rho}=0$, by considering the matrices $\tilde{A}_{i}:=A_{i}-\hat{\rho} I$.

(i) For ease of notation define

$$
\delta:=\min \left\{\left\|R^{-1}\right\|^{-1} \mid R \in \mathcal{S}_{\leq h}\right\}>0 .
$$

If the assertion is false, then there are $t_{k} \rightarrow \infty, S_{k} \in$ $\mathcal{S}_{t_{k}}\left(i_{k}\right)$ with $\left\|S_{k}\right\| \rightarrow \infty$. Without loss of generality we may assume, that $S_{k} \in \mathcal{R}_{t_{k}}\left(i_{k}, i_{k}\right)$. To see this, note that by Remark 3.1 we can always ensure, that $R_{k} S_{k} \in \mathcal{R}_{t_{k}}\left(i_{k}, i_{k}\right)$ for some $R_{k} \in \mathcal{S}_{h}$. It is easy to see, that $\left\|R_{k} S_{k}\right\| \geq$ $\left\|S_{k}\right\|\left\|R_{k}^{-1}\right\|^{-1} \geq\left\|S_{k}\right\| \delta \rightarrow \infty$ as $k \rightarrow \infty$.

Fix some $i \in\{1, \ldots, m\}$. The set $\mathcal{R}(i, i)$ is a semigroup and irreducible by Proposition 4.1. We may therefore use Lemma 4.1 to find constants $1 \geq \varepsilon_{1}>0$ and $T>0$, such that for all $x \in \mathbb{R}^{n}$ and all $B \in \mathbb{R}^{n \times n}$ there is an $R \in$ $\mathcal{R}_{\leq T}(i, i)$ with $\|B R x\| \geq \varepsilon_{1}\|B\|\|x\|$.

Now define $\varepsilon:=\min \left\{1, \varepsilon_{1} \delta^{2}\right\}$ and choose $k$ large enough such that

$$
\left\|S_{k}\right\|>4 / \varepsilon \text {. }
$$

Fix $U \in \mathcal{R}_{\leq 2 h}\left(i_{k}, i\right)$ and $V \in \mathcal{R}_{\leq 2 h}\left(i, i_{k}\right)$ and pick an arbitrary $x_{0} \in \mathbb{R}^{n},\left\|x_{0}\right\|=1$, such that $\left\|S_{k} x_{0}\right\| \geq\left\|S_{k}\right\| \varepsilon / 2$. Then we can choose $R_{1} \in \mathcal{R}_{\leq T}(i, i)$, such that

$$
\begin{array}{r}
\left\|S_{k} V R_{1} U S_{k} x_{0}\right\| \geq \varepsilon_{1}\left\|S_{k} V\right\|\left\|U S_{k} x_{0}\right\| \geq \\
\varepsilon_{1}\left\|S_{k}\right\|\left\|V^{-1}\right\|^{-1}\left\|U^{-1}\right\|^{-1}\left\|S_{k} x_{0}\right\| \geq\left(\left\|S_{k}\right\| \frac{\varepsilon}{2}\right)^{2} .
\end{array}
$$

Note that by construction $S_{k} V R_{1} U S_{k} \in$ $\mathcal{R}_{\leq 2 t_{k}+T+4 h}\left(i_{k}, i_{k}\right)$. Applying the same arguments again we can choose $R_{2} \in \mathcal{R}_{\leq T}(i, i)$, such that

$$
\left\|S_{k} V R_{2} U S_{k} V R_{1} U S_{k} x_{0}\right\| \geq\left(\left\|S_{k}\right\| \frac{\varepsilon}{2}\right)^{3} .
$$

Arguing inductively we construct times $\tau_{l}$ with $l t_{k} \leq \tau_{l} \leq$ $l\left(t_{k}+T+4 h\right)$ and matrices $T_{l} \in \mathcal{R}_{\tau_{l}}\left(i_{k}, i_{k}\right)$ with

$$
\begin{array}{r}
\frac{1}{\tau_{l}} \log \left\|T_{l}\right\| \geq \frac{l}{\tau_{l}} \log \left(\left\|S_{k}\right\| \frac{\varepsilon}{2}\right) \geq \frac{l}{\tau_{l}} \log 2 \\
\geq \frac{1}{t_{k}+T+4 h} \log 2>0 .
\end{array}
$$

This contradicts the assumption, that $\limsup _{l \rightarrow \infty} \frac{1}{\tau_{l}} \log \left\|T_{l}\right\| \leq 0$, which follows from $\hat{\rho}=0$.

(ii) A standard argument shows that $\mathcal{R}_{\infty}(i, j)$ is closed and by part (i) it is bounded. Thus we have to show, that there are nonzero elements. Now Lemma 3.3 shows, that there exists a constant $C>0$ and sequences $t_{k} \rightarrow \infty, S_{k} \in \mathcal{R}_{t_{k}}(i, j)$ with $\left\|S_{k}\right\| \geq C$ for all $k \in \mathbb{N}$. By (i) the sequence is bounded, so that it has a convergent subsequence with nonzero limit. By definition this limit is contained in $\mathcal{R}_{\infty}(i, j)$.

(iii) As $\mathcal{R}_{\infty}(i, j) \subset \mathcal{S}_{\infty}(i)$ it is clear from (i) that $\mathcal{S}_{\infty}(i)$ is nonempty and not equal to $\{0\}$. Closedness is immediate from the definition and so compactness follows from (i).

(iv) This is an easy exercise.

(v) Let $t_{k} \rightarrow \infty, u_{k} \in \mathcal{U}(i)$ be sequences such that $\Phi_{u_{k}}\left(t_{k}, 0\right) \rightarrow S \in \mathcal{S}_{\infty}(i)$. Applying Lemma 3.1 we may assume that there exists a $u \in \mathcal{U}(i)$ such that $\Phi_{u_{k}}(s, 0) \rightarrow$ $\Phi_{u}(s, 0)$, uniformly for $s \in[0,2 h]$. Thus we may write $\Phi_{u_{k}}\left(t_{k}, 0\right)=T_{k} \exp \left(A_{i} 2 h\right)$. Taking a convergent subsequence $T_{k} \rightarrow T$ shows the assertion.

(vi) Fix $i \in\{1, \ldots, m\}$. As we have noted the set $\mathcal{R}(i, i)$ is a semigroup, which is irreducible by Proposition 4.1. By (iv) it is easy to see that if $S \in \mathcal{R}(i, i) \cup \mathcal{R}_{\infty}(i, i)$, and $T \in \mathcal{R}_{\infty}(i, i)$ then $S T, T S \in \mathcal{R}_{\infty}(i, i)$ (where we have used the assumption $\hat{\rho}=0$, otherwise some further factors 
appear according to (iv)). Using (ii) this shows that $\mathcal{R}_{\infty}(i, i)$ is a nonzero semigroup ideal of the irreducible semigroup

$$
\mathcal{R}_{\infty}(i, i) \cup \mathcal{R}(i, i) .
$$

By [22, Lemma 1] this shows irreducibility of $\mathcal{R}_{\infty}(i, i)$. The second assertion follows from $\mathcal{R}_{\infty}(i, i) \subset \mathcal{S}_{\infty}(i)$.

The following interesting observation is obtained through the previous proof.

Corollary 5.1: Under the assumption of the previous Lemma 5.1 the set $\mathcal{S}(A, \mathcal{U})$ is bounded if $\hat{\rho}=0$.

Proof: If the assertion is false then there exists a sequence $\left\|S_{k}\right\| \rightarrow \infty$. This is brought to a contradiction in the proof of (i) of the previous theorem.

We now define for $i \in\{1, \ldots, m\}$ the function $v_{i}: \mathbb{R}^{n} \rightarrow$ $\mathbb{R}_{+}$by setting

$$
v_{i}(x):=\max \left\{\|S x\| \mid S \in \mathcal{S}_{\infty}(i)\right\} .
$$

Using Lemma 5.1 (iii) and (vi) it is easy to see, that for every $i \in\{1, \ldots, m\}$ the function defined in (14) is a norm on $\mathbb{R}^{n}$. The following result shows that in this manner we have defined a family of parameterized Lyapunov functions for our system.

Theorem 5.1: Let $\left\{A_{1}, \ldots, A_{m}\right\} \subset \mathbb{R}^{n \times n}$ be a finite irreducible set and let $h \in(0, \infty)$. Then the following two statements are equivalent

(i) $\hat{\rho}\left(A_{1}, \ldots, A_{m}, h\right) \leq \rho$,

(ii) there are norms $v_{1}, \ldots, v_{m}$ on $\mathbb{R}^{n}$ with the following properties:

$$
v_{i}\left(e^{A_{i} t} x\right) \leq e^{\rho t} v_{i}(x)
$$

for all $t \geq 0, x \in \mathbb{R}^{n}, i=1, \ldots, m$,

$$
v_{j}\left(e^{A_{j} t} x\right) \leq e^{\rho t} v_{i}(x)
$$

for all $t \geq h, x \in \mathbb{R}^{n}, i, j=1, \ldots, m$.

If the norms are defined through (14) then (15) and (16) hold with $\rho=\hat{\rho}\left(A_{1}, \ldots, A_{m}, h\right)$. Furthermore, for all $x \in \mathbb{R}^{n}$ and all $i \in\{1, \ldots, m\}$, there exists an $j \in\{1, \ldots, m\}$ and $t \in(0, h]$ such that

$$
v_{j}\left(\exp \left(A_{j} t\right) x\right)=e^{\hat{\rho} t} v_{i}(x) .
$$

Before proving the theorem we would like to point out the significance of (17). This equation states that at some bounded time instant where a switching may occur it is possible to exactly realize the exponential growth rate, with respect to the parameterized norms $v_{i}$. Note that this result is false if the assumption of irreducibility is violated. An easy example for this is given by a singleton set $\left\{A_{1}\right\}$. For $n>2$ a single matrix is never irreducible, and indeed the solutions of $\dot{x}=A x$ will in general display very different exponential growth behavior depending on the eigenvalues of $A$. If $x$ lies in an eigenspace corresponding to an eigenvalue $\lambda_{i}$ of $A$ the growth rate of $x$ can only be $\Re \lambda_{i}$.

Proof: (of Theorem 5.1) Without loss of generality we may assume $\hat{\rho}=0$.

(i) $\Rightarrow$ (ii): Define the norms $v_{i}$ through (14). For each $i \in$ $\{1, \ldots, m\}$ the function $u_{i}: \equiv i$ is contained in $\mathcal{U}(i)$. Assume that for some $x \in \mathbb{R}^{n} . t \geq 0$ we have $v_{i}\left(e^{A_{i} t} x\right) \geq v_{i}(x)$. Then by definition $\left\|T e^{A_{i} t} x\right\|>v_{i}(x)$ for some $T \in \mathcal{S}_{\infty}(i)$. Now Lemma 5.1 (iv) shows that $T e^{A_{i} t} \in \mathcal{S}_{\infty}(i)$. So that $v_{i}(x) \geq\left\|T e^{A_{i} t} x\right\|$, a contradiction.

A similar argument applies to the case (16), because $e^{A_{j} t} \in \mathcal{S}(i)$ for $t \geq h$. If we assume $v_{j}\left(e^{A_{j} t} x\right) \geq v_{i}(x)$, then we may choose $T \in \mathcal{S}_{\infty}(j)$ to realize the norm $v_{j}\left(e^{A_{j} t} x\right)=\left\|T e^{A_{j} t} x\right\|$ and again $T e^{A_{j} t} \in \mathcal{S}_{\infty}(i)$.

(ii) $\Rightarrow$ (i): It is sufficient to show that all Lyapunov exponents $\lambda(x, u)$ are upper bounded by $\rho$, [1]. So fix $0 \neq$ $x \in \mathbb{R}^{n}$ and an admissible switching signal $u$. If $u$ has no discontinuities on an interval of the form $(a, \infty)$, where $a \geq$ 0 , the assertion is obvious from (15). Otherwise let $t_{0}, t_{1}, \ldots$ denote the switching times of $u$ and let $i(k)$ be such that $u(t)=i(k)$, for $t \in\left[t_{k}, t_{k+1}\right)$. Without loss of generality let $t_{0}=0$, which we may assume as $\lambda(x, u)=\lambda\left(x, u\left(\cdot-t_{0}\right)\right)$. Then we have by (15), that

$$
v_{i(0)}\left(\exp \left(A_{i(0)} t\right) x\right) \leq e^{\rho t} v_{i(0)}(x), \quad \text { for } t \in\left[t_{0}+h, t_{1}\right],
$$

and so for $t \in\left[t_{1}+h, t_{2}\right]$ it follows, again using (16), that

$$
\begin{array}{r}
v_{i(1)}\left(\Phi_{u}(t, 0) x\right) \\
=v_{i(1)}\left(\exp \left(A_{i(1)}\left(t-t_{1}\right)\right) \exp \left(A_{i(0)} t_{1}\right) x\right) \\
\leq e^{\rho\left(t-t_{1}\right)} v_{i(0)}\left(\exp \left(A_{i(0)} t_{1}\right) x\right) \leq e^{\rho t} v_{i(0)}(x) .
\end{array}
$$

By induction we obtain for $t \in\left[t_{k}+h, t_{k+1}\right]$, that

$$
\frac{1}{t} \log \left(v_{i(k)}\left(\Phi_{u}(t, 0) x\right)\right) \leq \rho+\frac{1}{t} \log \left(v_{i(0)}(x)\right) .
$$

As the growth in the intervals $\left[t_{k}, t_{k}+h\right]$ is bounded, and as $v_{i(0)} \leq C v_{i}, i=1, \ldots, m$ for a suitable constant $C$, this implies, that $\lambda(x, u) \leq \rho$, as desired.

It remains to prove the final statement. Note that in the proof (i) $\Rightarrow$ (ii) we have already seen that for the norms defined in (14) the conditions (15) and (16) hold with $\rho=$ $\hat{\rho}\left(A_{1}, \ldots, A_{m}, h\right)$. So fix $x \in \mathbb{R}^{n}$ and $i \in\{1, \ldots, m\}$ and $t \geq 0$. Let $S \in \mathcal{S}_{\infty}(i)$ be such that $\|S x\|=v_{i}(x)$. By Lemma 5.1 (iv) there exist $j \in\{1, \ldots, m\}$ and $\exp \left(A_{j} t\right) \in$ $\mathcal{R}_{t}(i, j), T \in \mathcal{S}_{\infty}(\hat{j})$ such that $S=T \exp \left(A_{j} t\right)$ for a suitable $t \in(0, h]$. Then, using the definition of the norms $v_{i}$ and the equivalence (i) $\Leftrightarrow$ (ii) of this theorem, that we have just seen, we obtain

$$
\begin{aligned}
v_{i}(x) & =\|S x\|=\left\|T \exp \left(A_{j} h\right) x\right\| \\
& \leq v_{j}\left(\exp \left(A_{j} h\right) x\right) \leq v_{i}(x) .
\end{aligned}
$$

Thus equality holds throughout. This shows the assertion.

The previous result may also be formulated in differential terms. To this end we recall that the subgradient of a norm $\|\cdot\|$ at a point $x \neq 0$ is given by the normed dual vectors. The dual norm $\|\cdot\|_{*}$ is defined by

$$
\|y\|_{*}:=\max \{|\langle x, y\rangle| \mid\|x\| \leq 1\} .
$$

A vector $y$ is now a normed dual vector to $x$ if $\|y\|_{*}=1$ and

$$
\|x\|=\langle y, x\rangle .
$$


We denote the (convex) set of subgradient of the norm $\|\cdot\|$ in the point $x$ by

$$
\partial_{P}\|x\|:=\{y \mid y \text { is normed dual vector to } x\} .
$$

Corollary 5.2: Let $\left\{A_{1}, \ldots, A_{m}\right\} \subset \mathbb{R}^{n \times n}$ be a finite irreducible set and let $h \in(0, \infty)$. Then the following two statements are equivalent

(i) $\hat{\rho}\left(A_{1}, \ldots, A_{m}, h\right) \leq \rho$,

(ii) there are norms $v_{1}, \ldots, v_{m}$ on $\mathbb{R}^{n}$ with the following properties:

$$
\begin{aligned}
& \left\langle y, A_{i} x\right\rangle \leq \rho \quad \text { for all } \quad y \in \partial_{P} v_{i}(x), \\
& \left\langle y, A_{j} e^{A_{i}} x\right\rangle \leq \rho \quad \text { for all } \quad y \in \partial_{P} v_{i}(x),
\end{aligned}
$$

The norms may be chosen such that (19) and (20) hold with $\rho=\hat{\rho}\left(A_{1}, \ldots, A_{m}, h\right)$.

Proof: It is sufficient to show that (19) is equivalent to (15) and that (20) is equivalent to (16). This however is clear from general results of nonsmooth analysis.

Finally let us point out how the results generalize to reducible systems. We have already pointed out in (13) that in the reducible case, the matrices may be simultaneously transformed to upper block triangular form. With this form, the growth rate is determined by the blocks on the diagonal.

Corollary 5.3: Let $\left\{A_{1}, \ldots, A_{m}\right\} \subset \mathbb{R}^{n \times n}$ be a finite set and let $h \in(0, \infty)$. Then the following two statements are equivalent

(i) $\hat{\rho}\left(A_{1}, \ldots, A_{m}, h\right)<\rho$,

(ii) there are norms $v_{1}, \ldots, v_{m}$ on $\mathbb{R}^{n}$ with the following properties:

$$
v_{i}\left(e^{A_{i} t} x\right) \leq e^{\rho t} v_{i}(x)
$$

for all $t \geq 0, x \in \mathbb{R}^{n}, i=1, \ldots, m$,

$$
v_{j}\left(e^{A_{j} t} x\right) \leq e^{\rho t} v_{i}(x)
$$

for all $t \geq h, x \in \mathbb{R}^{n}, i, j=1, \ldots, m$.

Note that we have dropped the assumption of irreducibility and no statement of the form (17) can be made.

Proof: The proof (ii) $\Rightarrow$ (i) is just as in the proof of Theorem 5.1.

To prove the converse note that an easy calculation shows that the assumption implies that for each $i$ the set

$$
\left\{e^{-\rho t} \Phi_{u}(t, 0) \mid u \in \mathcal{U}(i)\right\}
$$

is bounded. We claim that the norms

$$
v_{i}(x):=\max \left\|e^{-\rho t} \Phi_{u}(t, 0)\right\|
$$

satisfy the assertion. This may be seen as follows: If $u \in$ $\mathcal{U}(i)$, then $\Phi_{u}(t, 0) \exp (A(i) t) \in \mathcal{S}(i)$. Then for all $t \geq 0$ and all $x \in \mathbb{R}^{n}$ we have

$$
\begin{array}{r}
v_{i}(\exp (A(i) t) x)=\max _{s, u \in \mathcal{U}(i)}\left\|e^{-\rho s} \Phi_{u}(s, 0) \exp (A(i) t) x\right\| \\
\leq e^{\rho t} v_{i}(x) .
\end{array}
$$

This shows (21) for the norms $v_{i}$. The second assertion can be shown by exactly the same argument applied to $\exp (A(j) t)$ for $t \geq h$.

\section{CONClusions}

A converse Lyapunov theorem has been presented for linear switching systems with dwell time. A particular feature is that in the generic irreducible case, the growth rate can be realized exactly at possible switching times. A more refined statement can be found in [1].

\section{ACKNOWLEDGEMENT}

This work was partially supported by Science Foundation Ireland under grant 00/PI.1/C067.

\section{REFERENCES}

[1] F. Wirth, "A converse Lyapunov theorem for linear parameter varying and linear switching systems," SIAM J. Control Optim., vol. 44, pp. 210-239, 2005.

[2] Y. Lin, E. D. Sontag, and Y. Wang, "A smooth converse Lyapunov theorem for robust stability," SIAM J. Control Optim., vol. 34, pp. 124-160, 1996.

[3] F. H. Clarke, Y. S. Ledyaev, and R. J. Stern, "Asymptotic stability and smooth Lyapunov functions," J. Differential Equations, vol. 149, no. 1, pp. 69-114, 1998.

[4] A. Teel and L. Praly, "A smooth Lyapunov function from a class$\mathcal{K} \mathcal{L}$ estimate involving two positive semidefinite function," ESAIM, Control Optim. Calc. Var, vol. 5, pp. 313-367, 2000.

[5] F. Wirth, "The Gelfand formula for linear parameter varying and linear switching systems." in Proc. 16th IFAC World Congress, Prague, Czech Republic, July 2005, pp. paper Th-M12-TO/6 (CD-Rom).

[6] — " "On Lipschitz continuity of the top Lyapunov exponent of linear parameter varying and linear switching systems," Stochastics \& Dynamics, vol. 4, no. 3, pp. 461-481, 2004.

[7] F. Colonius and W. Kliemann, "Maximal and minimal Lyapunov exponents of bilinear control systems," J. Diff. Eqns., vol. 101, pp. 232-275, 1993.

[8] — , "The Lyapunov spectrum of families of time varying matrices," Trans. Am. Math. Soc., vol. 348, pp. 4389-4408, 1996.

[9] - The Dynamics of Control. Boston: Birkhäuser, 2000.

[10] N. E. Barabanov, "Absolute characteristic exponent of a class of linear nonstationary systems of differential equations," Siberian Mathematical Journal, vol. 29, no. 4, pp. 521-530, 1988.

[11] A. P. Molchanov and E. S. Pyatnitskij, "Criteria of asymptotic stability of differential and difference inclusions encountered in control theory," Syst. Control Lett., vol. 13(1), pp. 59-64, 1989.

[12] F. Wirth, "The generalized spectral radius and extremal norms," Lin. Alg. Appl., vol. 342, pp. 17-40, 2002.

[13] W. P. Dayawansa and C. F. Martin, "A converse Lyapunov theorem for a class of dynamical systems which undergo switching," IEEE Trans. Automat. Control, vol. 44, no. 4, pp. 751-760, 1999.

[14] D. Liberzon and A. Morse, "Basic problems in stability design and design of switched systems," IEEE Control Systems Magazine, vol. 19, no. 5, pp. 59-70, 1999.

[15] D. Liberzon, Switching in systems and control, ser. Systems \& Control: Foundations \& Applications. Boston, MA: Birkhäuser Boston Inc., 2003.

[16] A. S. Morse, "Supervisory control of families of linear set-point controllers. I. Exact matching," IEEE Trans. Automat. Control, vol. 41, no. 10 , pp. 1413-1431, 1996.

[17] — , "Supervisory control of families of linear set-point controllers. II. Robustness," IEEE Trans. Automat. Control, vol. 42, no. 11, pp. 1500-1515, 1997.

[18] M. Johansson and A. Rantzer, "Computation of piecewise quadratic Lyapunov functions for hybrid systems," IEEE Trans. Automat. Control, vol. 43, no. 4, pp. 555-559, 1998.

[19] T. Kato, Perturbation Theory for Linear Operators, 2nd ed. Heidelberg: Springer-Verlag, 1980.

[20] U. Jönsson and A. Rantzer, "Systems with uncertain parameters time-variations with bounded derivative," Int. J. Robust \& Nonlinear Control, vol. 6(9/10), pp. 969-983, 1996.

[21] L. Elsner, "The generalized spectral-radius theorem: An analyticgeometric proof," Lin. Alg. Appl., vol. 220, pp. 151-159, 1995.

[22] H. Radjavi, "On irreducibility of semigroups of compact operators," Indiana Univ. Math. J., vol. 39, pp. 499-515, 1990. 